\title{
Machine Learning: A Novel Approach to Predicting Slope Instabilities
}

\author{
Upasna Chandarana Kothari $(\mathbb{D})$ and Moe Momayez $(\mathbb{D}$
}

Mining \& Geological Engineering, University of Arizona, Tucson, AZ, USA

Correspondence should be addressed to Upasna Chandarana Kothari; upasnap@gmail.com

Received 14 August 2017; Revised 16 January 2018; Accepted 18 January 2018; Published 20 February 2018

Academic Editor: Yun-tai Chen

Copyright (C) 2018 Upasna Chandarana Kothari and Moe Momayez. This is an open access article distributed under the Creative Commons Attribution License, which permits unrestricted use, distribution, and reproduction in any medium, provided the original work is properly cited.

\begin{abstract}
Geomechanical analysis plays a major role in providing a safe working environment in an active mine. Geomechanical analysis includes but is not limited to providing active monitoring of pit walls and predicting slope failures. During the analysis of a slope failure, it is essential to provide a safe prediction, that is, a predicted time of failure prior to the actual failure. Modernday monitoring technology is a powerful tool used to obtain the time and deformation data used to predict the time of slope failure. This research aims to demonstrate the use of machine learning (ML) to predict the time of slope failures. Twenty-two datasets of past failures collected from radar monitoring systems were utilized in this study. A two-layer feed-forward prediction network was used to make multistep predictions into the future. The results show an $86 \%$ improvement in the predicted values compared to the inverse velocity (IV) method. Eighty-two percent of the failure predictions made using ML method fell in the safe zone. While $18 \%$ of the predictions were in the unsafe zone, all the unsafe predictions were within five minutes of the actual failure time, all practical purposes making the entire set of predictions safe and reliable.
\end{abstract}

\section{Introduction}

Monitoring slope stability is an essential requirement in the field of geomechanics due to the potential threat a moving slope can cause to the workers or the business. Slope stability is an important concern for mining and civil engineers that deal with man-made slopes such as open-pit walls, dams, embankments of highways and railways, and hills. The causes of instability are often complex and creep theory is used in the design of rock slopes. The complexity of the causes of slope movement makes the time of slope failure prediction challenging. In recent years, the use of modern monitoring technologies has helped engineers better prepare for the outcomes of slope failures in open-pit mines [1].

Many attempts have been made to develop a method to predict the time of failure. Factors affecting slope instabilities such as ground conditions, physical and geomorphological processes, and human activities cannot be determined on a continuous basis, making it challenging to predict the time of slope failure accurately [2]. Hence, instead of developing a phenomenological model of slope failure, practitioners have relied on a detailed analysis of slope deformation [3].

Deformation data, the most relevant data for time series analysis of slope failures, is readily available from the monitoring equipment used for geotechnical risk management analysis $[4,5]$. Some of the modern and traditional monitoring technologies include but are not limited to tension crack mapping, survey networks, wireline extensometers, synthetic aperture radar, satellite-based synthetic aperture radar, and ground-based real aperture radar [6]. The radar systems usually record the increase in deformation accurately until the slope movement becomes too fast for the radar to capture or until slope collapses. The time and deformation data acquired from these monitoring systems will provide the opportunity to observe the prefailure evolution of a moving slope till the time of collapse. The three prefailure stages include primary, secondary, and tertiary movement (Figure 1). The primary stage displays a decreasing strain rate, the secondary stage displays a constant strain rate, and the tertiary stage represents an accelerating strain rate leading to 


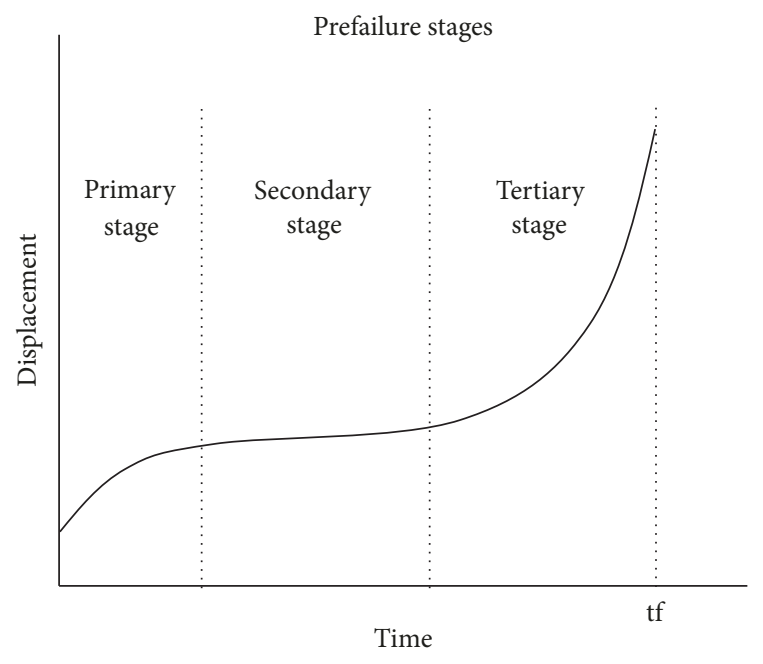

Figure 1: Primary stages of prefailure evolution.

failure. The prefailure evolution of slope movement exhibits similar characteristics to the creep observed in the study of geomaterials [7-10].

Based on the core understanding of the prefailure evolution, many attempts have been made to develop suitable methods to predict an accurate time of slope failure. Many of the slope failure studies have used the inverse velocity (IV) method proposed by Fukuzono in 1985 [11]. The fuzzy neural network approach is another method that gained popularity in the civil engineering industry and was slowly adapted for slope stability analysis. Predicting slope failure is a common practice in active mines to prevent injuries and fatalities due to ground movement issues. With a view to make a prediction that allows for ample evacuation time, the forecast should provide a time before the actual slope failure. As all operations are different, the time required to evacuate the area will solely depend on the size of the mine and the resources available. A prediction that occurs before the actual time of failure is considered a safe prediction, whereas a forecast that occurs after the actual time of failure would be regarded as an unsafe prediction. It is therefore highly desirable to make a safe failure prediction to evacuate the area if required.

The aim of this paper is to investigate the use of machine learning (ML) to make safe predictions. Mitchell defined machine learning as the question of how to build computer programs that improve their performance at some task through experience in 1997 [12]. Machine learning enables the computer to recognize patterns and explore the data and uses algorithms to help make predictions based on the input data. There are many algorithms available today to sort through the data, learn visible and invisible patterns, and use the learnt pattern to make better decisions. There are three main types of machine learning, namely, supervised learning, unsupervised learning, and semisupervised learning. The three types of machine learning are briefly explained below:

(i) Supervised learning: a set of data with the known solution is used as the input data; the input data is called training data. The training data is used to train

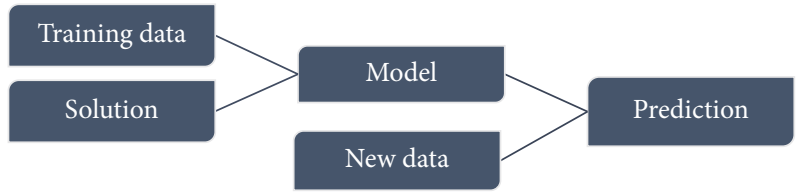

FIGURE 2: Flow chart of supervised machine learning.

the computer to learn trends and build a model to make informed predictions. Corrections are made to the model for the training process till the desired results are achieved. Classification and regression are examples of supervised learning [13].

(ii) Unsupervised learning: a set of data with unknown solution is used as the input data. For unsupervised learning, a model is built by assuming the presence of structures in the input data by looking for redundancy or similarity in the data [13].

(iii) Semisupervised learning: dataset is a mixture of known and unknown solutions. For this learning, the model is built to understand structure and make predictions [13].

The application of the fuzzy neural network in slope stability studies is an example of supervised ML. The fuzzy set theory has been used in the past to analyze the potential of a slope failure. These studies successfully demonstrated how the fuzzy neural network could assist preparing for a potential slope failure; however, the fuzzy neural networks have not been used for predicting slope failure [14-18]. Fuzzy set theory is a machine learning system based on the real-life model of the neuron's work in a human brain. In 1965, Zadeh first introduced the fuzzy set theory, which was adopted for analysis that can be probabilistic or deterministic [19].

The primary goal of slope failure analysis is to predict the time of slope failure in the presence of evidence that demonstrates signs of a possible slope failure. Similarly, the aim of supervised machine learning is to build a model that can make predictions based on evidence in the data. Using adaptive algorithms, the prediction network learns from the training data and builds a model that is used to make predictions. A large set of training data provides more observations for the training set to learn from and improve its predictive performance. Figure 2 presents a flow chart of how supervised machine learning is used in this study. The idea to attempt a machine learning approach was inspired by a previous study conducted by the authors, where they proposed the use of minimum inverse velocity (MIV) method to improve the accuracy of slope failure predictions [20].

\section{Methodology}

The approach we propose to predict the time of failure is based on nonlinear regression using a two-layer feedforward network. A feed-forward network is a unit in which the processes would not form a cycle; the processes would flow from start to finish like a chain reaction (Figure 2). 


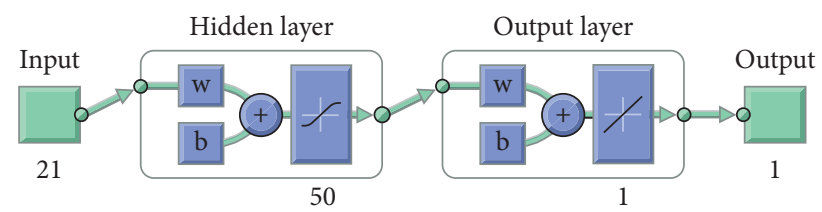

FIgURE 3: Flow chart of the prediction network.

After preprocessing the 22 datasets at our disposal, the timedeformation data is divided into training, validation, and test sets, a network architecture is selected, the network is trained, and multistep prediction is performed. The prediction network was designed in MATLAB using the Neural Network Toolbox. Figure 3 shows the structure of the network.

The datasets collected at open-pit mining operations have different sampling intervals and deformation rates. To obtain the best predictions, preprocessing must be performed to standardize the time series. This step consists of resampling and rescaling the data. Resampling involves changing the sampling interval in each time-deformation series to match or set to a value less than the smallest sampling interval among all the datasets. In this study, a linear interpolation method was used. The deformation data from different mine sites have a wide range of values. When the testing was initiated, the trial tests confirmed that the prediction network is sensitive to magnitude because features with larger values impose more influence on the training set. Transforming data, to have values between 0 and 1 , considerably improved the accuracy of predictions.

As mentioned above, this research has been inspired by a recent study conducted by the authors [20], where they introduced the concept of the minimum inverse velocity. MIV was shown to improve the time of failure predictions compared to the traditional IV method proposed by Fukuzono in 1985 [11]. The predictions from the MIV were obtained using data from a two-hour time window before slope failure. In the machine learning study, we predicted deformation for the same twohour window to compare the performances of the ML and MIV methods.

To predict the failure time in each deformation curve, we created an eight-hour training set consisting of the resampled and rescaled data from the other 21 datasets. Different algorithms that update the weight and bias values in the network training function were tested. We settled on the Levenberg-Marquardt algorithm, since it provides the fastest convergence and better overall prediction values. The property DIVIDEMODE was set to TIMESTEP forcing the targets to be divided into training, validation, and test sets according to timesteps. Various segmentations of training, validation, and test sets were tried, and no significant impact on the final results was observed. For the remainder of the study, $70 \%$ of training, $15 \%$ of validation, and $15 \%$ of testing data were specified from the total dataset of 22 available records.

A curvature index (the normalized area between the time-deformation curve and a straight line connecting the first point in the time series to the failure point) was calculated for each of the 22 datasets used in this research.
12 time series exhibited a linear type deformation, curvature index close to zero. Nine datasets had a curvature index less than -0.1 , representing a regressive type deformation. One dataset had a curvature index greater than +0.1 , indicative of a progressive deformation. To perform a multistep prediction for a given dataset, the values for the last two-hour window in the time-deformation series were set to $\mathrm{NaN}$ (not a number). After much experimentation, we settled on a value of 50 nodes in the hidden layer as it provided the smallest number of nodes and most consistent prediction values.

During training, the network performance over 250 to 400 timesteps produced a mean square error of less than $10^{-5}$ as shown in Figure 4. In Figure 4, the $x$-axis represents the error and the Zero Error line represents the mean square error, whereas the $y$-axis represents the number of instances the algorithm ran in order to produce the results. Next, the first peak in the output sequence of the network was used to determine the time of failure for the slope. Figure 5 provides a plot of the original dataset and the predicted values for mine site 20 .

\section{Results}

The predictions obtained from the MIV resulted in a 75\% improvement in comparison to the IV method [20]. Table 1 presents a summary of the results comparing the IV and MIV methods including the predicted time of failures. The predictions based on both methods have been compared to the real time of failure. The negative values in the column "IVMIV" represent a success (prediction in the safe zone) for the IV method whereas the positive values represent a success for the MIV method. Based on the results below, we can see that MIV method results in a $75 \%$ improvement in slope failure predictions.

Some machine learning algorithms perform well when used for fitting data especially if the training set contains strong features. The curvature indices in the 22 datasets range from -0.324 to +0.127 with most of the deformation curves displaying a linear behavior (a curvature index close to zero). Because the time series in the training set have a relatively similar form, we surmised that ML would provide prediction values that are closer to the real time of failure. Twenty-two historical failure datasets with a time span of 8 hours were used to generate the training dataset. Table 2 summarizes the results obtained using ML to predict the time of failure. All the predictions were made based on two hours of missing data prior to the failure. The results of ML are compared to the traditional IV method. The column "IV-ML" provides the time difference between the two approaches. Positive values represent the number of hours ML prediction is closer to the real time of failure compared to IV. Each positive value in the "IV-ML" column represents a success for ML, whereas each negative value represents a success for IV. Based on the results, 19 cases demonstrate a better prediction. The results show an $86.4 \%$ success rate in the predictions obtained using the ML method.

The predictions obtained using ML method for the slope failure were compared to the results based on the MIV method. Table 3 shows a comparison between MIV and 
TABLE 1: Results of the comparison between inverse velocity (IV) method and minimum inverse velocity (MIV) method from 22 different failure examples. For the analysis demonstrated below, all calculations are based on a 60-minute averaging window.

\begin{tabular}{|c|c|c|c|c|c|c|}
\hline \# & Actual time of failure & $\begin{array}{l}\text { Prediction: } \\
\text { IV }\end{array}$ & $\begin{array}{c}\text { Delta time } \\
(\mathrm{hr})\end{array}$ & $\begin{array}{l}\text { Prediction: } \\
\text { MIV }\end{array}$ & $\begin{array}{c}\text { Delta time } \\
(\mathrm{hr})\end{array}$ & $\begin{array}{c}\text { IV - MIV } \\
(\mathrm{hr})\end{array}$ \\
\hline (1) & 3/10/09 7:12 & $3 / 10 / 097: 51$ & 0.65 & $3 / 10 / 097: 42$ & 0.52 & 0.13 \\
\hline (2) & 3/13/13 9:48 & $3 / 13 / 139: 19$ & -0.48 & 3/13/13 8:45 & -1.04 & -1.51 \\
\hline (3) & $3 / 5 / 124: 09$ & $3 / 5 / 124: 34$ & 0.43 & $3 / 5 / 124: 31$ & 0.38 & 0.05 \\
\hline (4) & $8 / 3 / 1322: 06$ & $8 / 3 / 1323: 53$ & 1.79 & 8/3/13 22:15 & 0.16 & 1.63 \\
\hline (5) & $7 / 27 / 1218: 50$ & $7 / 27 / 1219: 38$ & 0.81 & 7/27/12 19:00 & 0.18 & 0.64 \\
\hline (6) & $10 / 24 / 1322: 39$ & $10 / 24 / 1322: 40$ & 0.03 & $10 / 24 / 1320: 58$ & -1.68 & -1.65 \\
\hline (7) & 5/5/14 5:04 & $5 / 6 / 142: 41$ & 21.62 & $5 / 4 / 1420: 23$ & -8.67 & 12.96 \\
\hline (8) & 6/16/14 6:58 & $6 / 16 / 147: 10$ & 0.21 & 6/16/14 4:01 & -2.95 & -2.74 \\
\hline (9) & 1/28/12 9:14 & 1/28/12 12:04 & 2.84 & 1/28/12 9:08 & -0.10 & 2.74 \\
\hline (10) & $10 / 29 / 1212: 15$ & $10 / 31 / 1218: 58$ & 54.73 & $10 / 29 / 1211: 58$ & -0.28 & 54.45 \\
\hline (11) & $9 / 25 / 128: 40$ & $9 / 26 / 12$ 18:16 & 33.61 & $9 / 25 / 124: 33$ & -4.12 & 29.49 \\
\hline (12) & $3 / 26 / 1321: 03$ & 4/10/13 23:08 & 362.10 & $3 / 26 / 1323: 58$ & 2.92 & 359.18 \\
\hline (13) & $2 / 24 / 1211: 27$ & $2 / 24 / 1217: 03$ & 5.61 & $2 / 24 / 1213: 42$ & 2.26 & 3.36 \\
\hline (14) & $3 / 2 / 144: 23$ & $3 / 2 / 145: 55$ & 1.54 & $3 / 2 / 145: 48$ & 1.42 & 0.12 \\
\hline (15) & $10 / 25 / 1216: 17$ & $10 / 25 / 1216: 47$ & 0.51 & $10 / 25 / 1215: 35$ & -0.69 & -0.18 \\
\hline (16) & $4 / 22 / 1313: 27$ & $4 / 22 / 1313: 58$ & 0.52 & 4/22/13 10:02 & -3.40 & -2.88 \\
\hline (17) & $3 / 14 / 104: 01$ & $3 / 14 / 104: 16$ & 0.26 & $3 / 14 / 104: 02$ & 0.03 & 0.24 \\
\hline (18) & 7/11/13 0:20 & $7 / 11 / 130: 25$ & 0.10 & $7 / 11 / 130: 13$ & -0.11 & -0.01 \\
\hline (19) & 7/21/11 15:01 & $7 / 21 / 1115: 17$ & 0.28 & 7/21/11 14:35 & -0.42 & -0.14 \\
\hline (20) & $6 / 20 / 0922: 24$ & $6 / 21 / 09$ 1:09 & 2.76 & $6 / 20 / 0923: 58$ & 1.57 & 1.20 \\
\hline (21) & 2/9/10 12:38 & 2/9/10 14:05 & 1.47 & 2/9/10 13:20 & 0.71 & 0.75 \\
\hline (22) & $1 / 30 / 1518: 25$ & $1 / 30 / 1521: 55$ & 3.51 & 1/30/15 21:11 & 2.78 & 0.74 \\
\hline
\end{tabular}

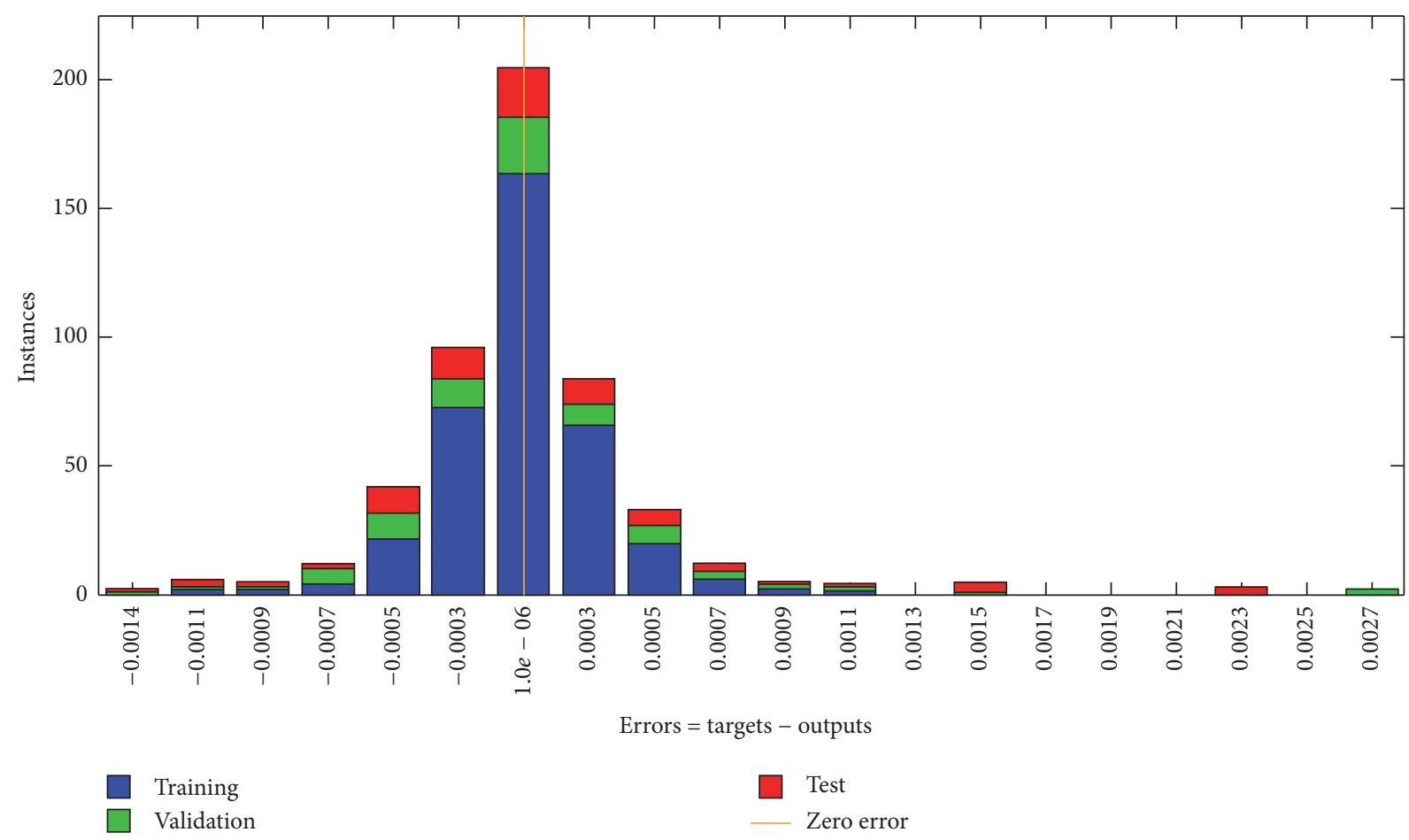

FIGURE 4: Error histogram from a training period. 
TABLE 2: Results of the comparison between inverse velocity (IV) method and machine learning (ML) method from 22 different failure examples.

\begin{tabular}{|c|c|c|c|c|c|c|}
\hline \# & Actual time of failure & $\begin{array}{l}\text { Prediction: } \\
\text { IV }\end{array}$ & $\begin{array}{c}\text { Delta time } \\
(\mathrm{hr})\end{array}$ & $\begin{array}{c}\text { Prediction: } \\
\text { ML }\end{array}$ & $\begin{array}{c}\text { Delta time } \\
(\mathrm{hr})\end{array}$ & $\begin{array}{c}\text { IV }-\mathrm{ML} \\
(\mathrm{hr})\end{array}$ \\
\hline (1) & 3/10/09 7:12 & 3/10/09 7:51 & 0.65 & $3 / 10 / 096: 17$ & -0.92 & -0.27 \\
\hline (2) & 3/13/13 9:48 & 3/13/13 9:19 & -0.48 & 3/13/13 9:52 & 0.07 & 0.41 \\
\hline (3) & 3/5/12 4:09 & $3 / 5 / 124: 34$ & 0.43 & $3 / 5 / 123: 24$ & -0.75 & -0.32 \\
\hline (4) & 8/3/13 22:06 & $8 / 3 / 1323: 53$ & 1.79 & 8/3/13 21:56 & -0.17 & 1.62 \\
\hline (5) & $7 / 27 / 1218: 50$ & $7 / 27 / 1219: 38$ & 0.81 & $7 / 27 / 1218: 51$ & 0.02 & 0.79 \\
\hline (6) & $10 / 24 / 1322: 39$ & $10 / 24 / 1322: 40$ & 0.03 & $10 / 24 / 1322: 40$ & 0.02 & 0.01 \\
\hline (7) & 5/5/14 5:04 & 5/6/14 2:41 & 21.62 & 5/5/14 4:13 & -0.85 & 20.77 \\
\hline (8) & $6 / 16 / 146: 58$ & 6/16/14 7:10 & 0.21 & 6/16/14 6:57 & -0.02 & 0.19 \\
\hline (9) & 1/28/12 9:14 & $1 / 28 / 1212: 04$ & 2.84 & 1/28/12 8:19 & -0.92 & 1.92 \\
\hline (10) & $10 / 29 / 1212: 15$ & $10 / 31 / 1218: 58$ & 54.73 & 10/29/12 11:59 & -0.27 & 54.46 \\
\hline (11) & $9 / 25 / 128: 40$ & $9 / 26 / 1218: 16$ & 33.61 & $9 / 25 / 128: 44$ & 0.07 & 33.54 \\
\hline (12) & $3 / 26 / 1321: 03$ & 4/10/13 23:08 & 362.10 & $3 / 25 / 1319: 40$ & -1.38 & 360.72 \\
\hline (13) & 2/24/12 11:27 & 2/24/12 17:03 & 5.61 & $2 / 24 / 1211: 25$ & -0.03 & 5.58 \\
\hline (14) & $3 / 2 / 144: 23$ & $3 / 2 / 145: 55$ & 1.54 & $3 / 2 / 144: 22$ & -0.02 & 1.52 \\
\hline (15) & 10/25/12 16:17 & $10 / 25 / 1216: 47$ & 0.51 & $10 / 25 / 1215: 24$ & -0.88 & -0.37 \\
\hline (16) & $4 / 22 / 1313: 27$ & $4 / 22 / 1313: 58$ & 0.52 & 4/22/13 13:31 & 0.07 & 0.45 \\
\hline (17) & $3 / 14 / 104: 01$ & $3 / 14 / 104: 16$ & 0.26 & $3 / 14 / 103: 50$ & -0.18 & 0.08 \\
\hline (18) & 7/11/13 0:20 & 7/11/13 0:25 & 0.10 & 7/11/13 0:19 & -0.02 & 0.08 \\
\hline (19) & 7/21/11 15:01 & 7/21/11 15:17 & 0.28 & 7/21/11 15:00 & -0.02 & 0.26 \\
\hline (20) & $6 / 20 / 09$ 22:24 & 6/21/09 1:09 & 2.76 & $6 / 20 / 0922: 23$ & -0.02 & 2.74 \\
\hline (21) & 2/9/10 12:38 & 2/9/10 14:05 & 1.47 & 2/9/10 12:30 & -0.13 & 1.34 \\
\hline$(22)$ & 1/30/15 18:25 & 1/30/15 21:55 & 3.51 & 1/30/15 16:34 & -1.85 & 1.66 \\
\hline
\end{tabular}

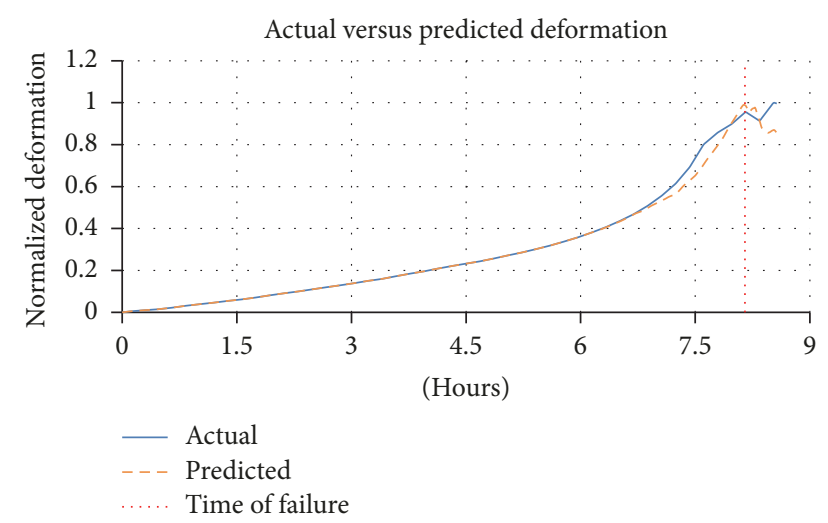

FIGURE 5: Actual and predicted slope failure data from mine site 20.

ML methods. The column "MIV-ML" provides the time difference between the two approaches. A negative value in the MIV-ML value represents a success (prediction in the safe zone) for the MIV method whereas all the positive values represent a success for the ML method. A comparison of the MIV and ML methods provides a $72 \%$ success rate for the machine learning technique. Out of the 22 cases studies, $\mathrm{ML}$ method gave better results in 16 cases. Based on the overall results, ML performed significantly better than IV and MIV methods.
After comparing the three methods, IV, MIV, and ML, it is concluded that ML gives the results that are the closest to the real time of failure. A $95 \%$ confidence interval was calculated for the three methods. The results are displayed in Table 4. Based on the confidence interval calculations, it is concluded that $95 \%$ of the slope failure predictions using the IV method will fall between -131 and 176 hours from the real time of failure. As the confidence level was applied to the datasets used for the analysis, 21 of the prediction fell in the $95 \%$ confidence interval using IV method. The confidence interval calculated for MIV indicates that 95\% of the slope failure predictions calculated using MIV will fall between -6 and 5 hours away from the real time of failure. Twenty-one of the 22 datasets analyzed gave a failure prediction that fell into the $95 \%$ confidence interval with the MIV method. The confidence interval calculated for ML indicates that $95 \%$ of the slope failure predictions calculated using ML will fall between -1.45 and 0.72 hours away from the real time of failure. Twenty-one of the 22 datasets analyzed using ML method gave a failure prediction that fell in the $95 \%$ confidence interval. In addition to giving the best results, ML also has the smallest time window between the lower and upper limit of the $95 \%$ confidence interval.

In addition to getting a prediction time that is close to the real time of failure, another aim of this study was to provide a time of failure prediction that falls in the safe 
TABLE 3: Results of the comparison between minimum inverse velocity (MIV) and machine learning (ML) method from 22 different failure examples.

\begin{tabular}{|c|c|c|c|c|c|c|}
\hline \# & Actual time of failure & $\begin{array}{l}\text { Prediction: } \\
\text { MIV }\end{array}$ & $\begin{array}{c}\text { Delta time } \\
(\mathrm{hr})\end{array}$ & $\begin{array}{l}\text { Prediction: } \\
\text { ML }\end{array}$ & $\begin{array}{l}\text { Delta time } \\
(\mathrm{hr})\end{array}$ & $\begin{array}{c}\text { MIV - ML } \\
\text { (hr) }\end{array}$ \\
\hline (1) & $3 / 10 / 09$ 7:12 & $3 / 10 / 097: 42$ & 0.52 & 3/10/09 6:17 & -0.92 & -0.40 \\
\hline (2) & 3/13/13 9:48 & $3 / 13 / 138: 45$ & -1.04 & 3/13/13 9:52 & 0.07 & 0.97 \\
\hline (3) & 3/5/12 4:09 & $3 / 5 / 124: 31$ & 0.38 & $3 / 5 / 123: 24$ & -0.75 & -0.37 \\
\hline (4) & 8/3/13 22:06 & 8/3/13 22:15 & 0.16 & 8/3/13 21:56 & -0.17 & -0.01 \\
\hline (5) & $7 / 27 / 1218: 50$ & $7 / 27 / 12$ 19:00 & 0.18 & $7 / 27 / 1218: 51$ & 0.02 & 0.16 \\
\hline (6) & $10 / 24 / 1322: 39$ & $10 / 24 / 1320: 58$ & -1.68 & $10 / 24 / 1322: 40$ & 0.02 & 1.66 \\
\hline (7) & 5/5/14 5:04 & 5/4/14 20:23 & -8.67 & 5/5/14 4:13 & -0.85 & 7.82 \\
\hline (8) & 6/16/14 6:58 & 6/16/14 4:01 & -2.95 & 6/16/14 6:57 & -0.02 & 2.93 \\
\hline (9) & 1/28/12 9:14 & 1/28/12 9:08 & -0.10 & 1/28/12 8:19 & -0.92 & -0.82 \\
\hline (10) & $10 / 29 / 12$ 12:15 & 10/29/12 11:58 & -0.28 & 10/29/12 11:59 & -0.27 & 0.01 \\
\hline (11) & $9 / 25 / 128: 40$ & $9 / 25 / 124: 33$ & -4.12 & 9/25/12 8:44 & 0.07 & 4.05 \\
\hline (12) & $3 / 26 / 13$ 21:03 & $3 / 26 / 13$ 23:58 & 2.92 & $3 / 25 / 13$ 19:40 & -1.38 & 1.54 \\
\hline (13) & 2/24/12 11:27 & $2 / 24 / 1213: 42$ & 2.26 & 2/24/12 11:25 & -0.03 & 2.23 \\
\hline (14) & $3 / 2 / 144: 23$ & $3 / 2 / 145: 48$ & 1.42 & $3 / 2 / 144: 22$ & -0.02 & 1.40 \\
\hline (15) & 10/25/12 16:17 & $10 / 25 / 1215: 35$ & -0.69 & $10 / 25 / 1215: 24$ & -0.88 & -0.19 \\
\hline (16) & $4 / 22 / 1313: 27$ & 4/22/13 10:02 & -3.40 & $4 / 22 / 1313: 31$ & 0.07 & 3.33 \\
\hline (17) & 3/14/10 4:01 & 3/14/10 4:02 & 0.03 & $3 / 14 / 103: 50$ & -0.18 & -0.15 \\
\hline (18) & 7/11/13 0:20 & 7/11/13 0:13 & -0.11 & 7/11/13 0:19 & -0.02 & 0.09 \\
\hline (19) & 7/21/11 15:01 & 7/21/11 14:35 & -0.42 & 7/21/11 15:00 & -0.02 & 0.40 \\
\hline (20) & $6 / 20 / 09$ 22:24 & $6 / 20 / 09$ 23:58 & 1.57 & $6 / 20 / 09$ 22:23 & -0.02 & 1.55 \\
\hline (21) & 2/9/10 12:38 & 2/9/10 13:20 & 0.71 & 2/9/10 12:30 & -0.13 & 0.58 \\
\hline$(22)$ & $1 / 30 / 1518: 25$ & 1/30/15 21:11 & 2.78 & 1/30/15 16:34 & -1.85 & 0.93 \\
\hline
\end{tabular}

TABLE 4: Confidence interval calculated for the IV and MIV methods. The calculations use $\mu \pm 2 \sigma$ to get the upper and lower bounds of the $95.5 \%$ confidence interval.

\begin{tabular}{lccc}
\hline \multicolumn{4}{c}{ 95.5\% confidence interval } \\
& IV method & MIV method & ML method \\
\hline Mean $(\mu)$ & 22.5 & -0.48 & -0.37 \\
Standard deviation $(\sigma)$ & 77.04 & 2.57 & 0.54 \\
Upper limit & 176.52 & 4.66 & 0.72 \\
Lower limit & -131.39 & -5.62 & -1.46 \\
\hline
\end{tabular}

zone. Figures 6-8 demonstrate the distribution of the failure prediction using IV, MIV, and ML method. The distribution of the time of failure predictions is compared to the real time of failure, distinguishing the predictions as safe or unsafe. A failure prediction is considered a safe prediction when the failure occurs after the predicted time, whereas if the failure occurs before the predicted time, it is deemed to be an unsafe prediction. In the figures demonstrating the prediction distribution, line $\mathrm{AB}$ represents the life expectancy of the moving slope; any prediction below line $A B$ is a safe prediction whereas any prediction above line $A B$ is considered an unsafe prediction. The results were rotated 45 degrees and plotted on an $x-y$ plot to distinguish between safe and unsafe predictions. In Figures 6-8, let us assume that the

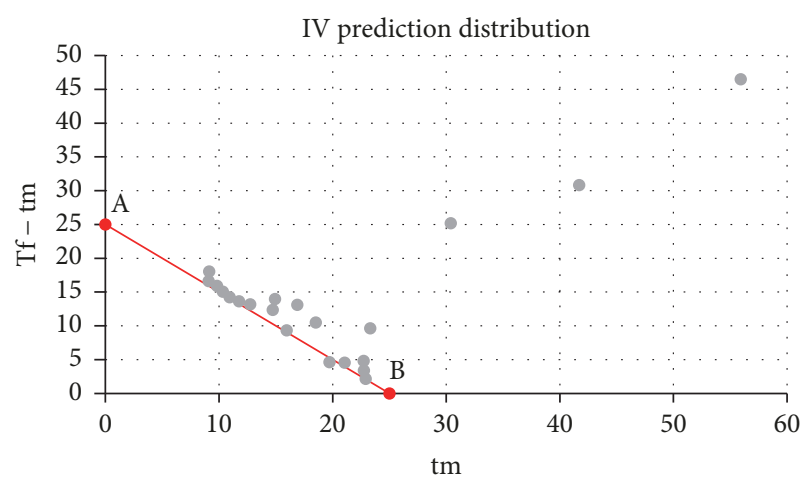

FIGURE 6: Distribution of failure predictions using IV method. Line $\mathrm{AB}$ represents failure time.

$x$-axis represents the predicted time of failure whereas the $y$ axis represents the actual time of failure minus the predicted time.

Tables 1-3 provide all the results using IV, MIV, and ML method to predict the time of failure. The results are represented in a graphical format in Figures 6-8. Figure 6 represents the prediction distribution of the IV method; the outlier with a time difference of 362 hours was eliminated from the graph to demonstrate a better visualization of the rest of the data. Figure 6 shows that some of the predictions 
TABLE 5: Comparison between failure predictions 1, 2, 3, 4, and 5 hours prior to failure for locations 8 and 20.

\begin{tabular}{lcccccc}
\hline \multirow{2}{*}{ Location } & \multirow{2}{*}{ Failure time } & \multicolumn{4}{c}{ Prediction: 1, 2, 3, 4, and 5 hours before failure } \\
& & 1 & 2 & 3 & 4 & 5 \\
\hline \multirow{2}{*}{08} & $\mathbf{6 / 1 6 / 1 4} \mathbf{6 : 5 8}$ & $6 / 16 / 147: 01$ & $6 / 16 / 146: 57$ & $6 / 16 / 145: 39$ & $6 / 16 / 144: 51$ & $6 / 16 / 146: 26$ \\
& Time difference & 0.05 & -0.02 & -1.37 & -2.20 & -0.56 \\
\hline \multirow{2}{*}{20} & $\mathbf{6 / 2 0 / 0 9 2 2 : 2 4}$ & $6 / 20 / 0922: 17$ & $6 / 20 / 0922: 23$ & $6 / 20 / 0921: 48$ & $6 / 20 / 0921: 46$ & $6 / 20 / 0921: 28$ \\
& Time difference & -0.12 & -0.02 & -0.60 & -0.63 & -0.93 \\
\hline
\end{tabular}

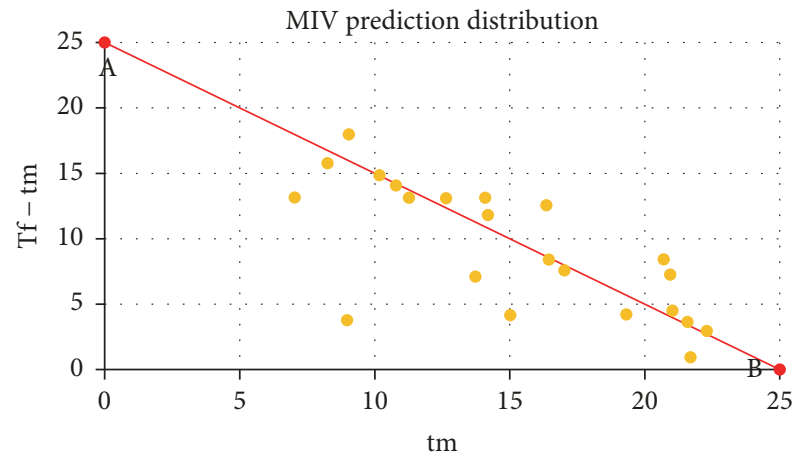

Figure 7: Distribution of failure predictions using MIV method. Line $A B$ represents failure time.

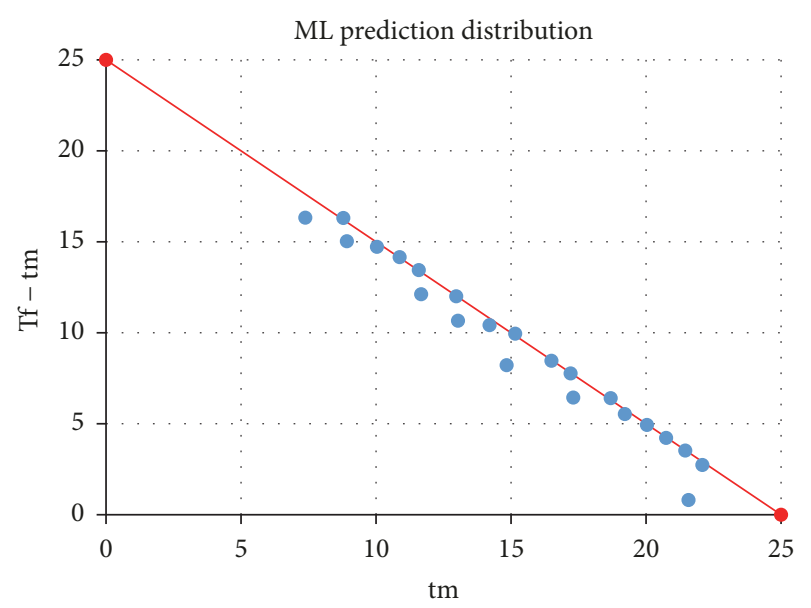

FIGURE 8: Distribution of failure predictions using ML method. Line $\mathrm{AB}$ represents failure time.

are close to the real time of failure, but there is only one safe prediction. Figure 7 represents the distribution of MIV method, demonstrating that $50 \%$ of the failure predictions are in the safe zone. Figure 8 represents the distribution of ML method; this graph shows that all the predictions are very close to the real time of failure. It is hard to see from the graph but only 4 of the predictions using ML method occur after the actual failure time and all the unsafe predictions are within a 5-minute interval from the real time of failure and can be considered as a safe prediction. Statistically, $82 \%$ of the predictions using ML fall in the safe zone. The comparisons between IV, MIV, and ML show a significant improvement in the time of failure predictions using the ML method.
Machine learning analysis was also used to analyze the accuracy of the failure predictions with respect to the proximity of the real time of failure to current data. Two datasets were chosen from the twenty-two records utilized in this study, and predictions were made for time series $1,2,3$, 4 , and 5 hours before the failure. This analysis showed that predictions improve as the actual time of failure approaches. Theoretically, slope failure predictions should get closer to the real time of failure as the size of the collected dataset increases. When an accelerating movement is observed in the data, the trend is defined as progressive or regressive as time goes on. If predictions are made with a well-defined progressive curve, the chance of a better prediction improves as the time of actual failure approaches. The investigation related to making predictions $1,2,3,4$, and 5 hours prior to failure confirmed the above hypothesis. Results in Table 5 show a trend of decreasing time difference as the real time of failure approaches.

From the two datasets analyzed, it can be concluded that failure predictions made two hours before the failure are the closest to the time of failure, resulting in the best time of failure predictions. In general, as a slope approaches failure, the rate of deformation increases rapidly. If the rate of slope movement is faster than the scan rate, it is likely that the monitoring system does not capture the entire deformation. When the radar is not able to record the movement correctly, the time-deformation curve appears to drop or to slow down. Due to this limitation of the monitoring systems, it has been observed that the gap between the predicted and actual failure time increases.

\section{Discussion}

Risk identification, risk management, and risk mitigation processes benefit from reliable slope stability monitoring and forecasting. The desired outcome of slope stability monitoring is to be able to make safe predictions. Any prediction that occurs before the actual failure time is considered a safe prediction. ML approach resulted in 17 failure predictions that occurred in the safe zone and five predictions that were in the unsafe zone. The five unsafe predictions were within 5 minutes of the real time of failure. Therefore, for all practical purposes, they could be considered as safe predictions.

The selection of alarm thresholds at a mine site is often based on historical behavior of slopes and could present challenges as one or several factors controlling slope deformation change suddenly. Increasing the scan rate can help improve the reliability of capturing unexpected acceleration. However, in many situations, due to the large distance from the face 


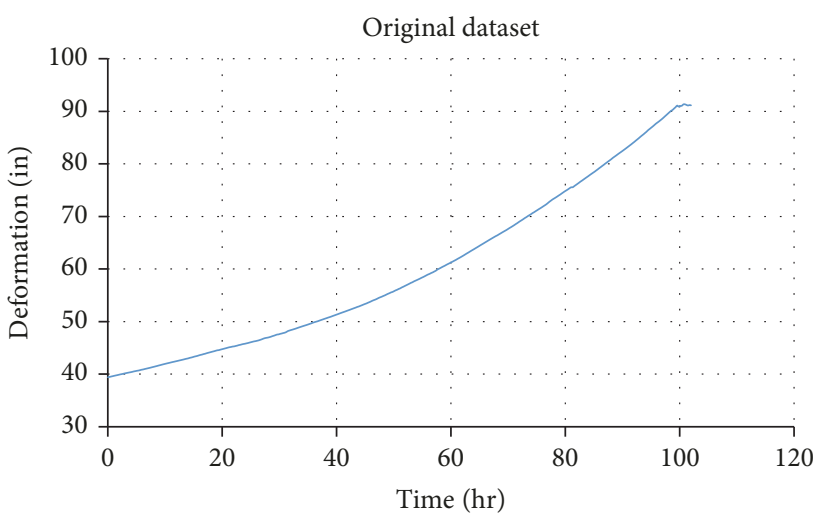

(a) Original dataset shows progressive trend

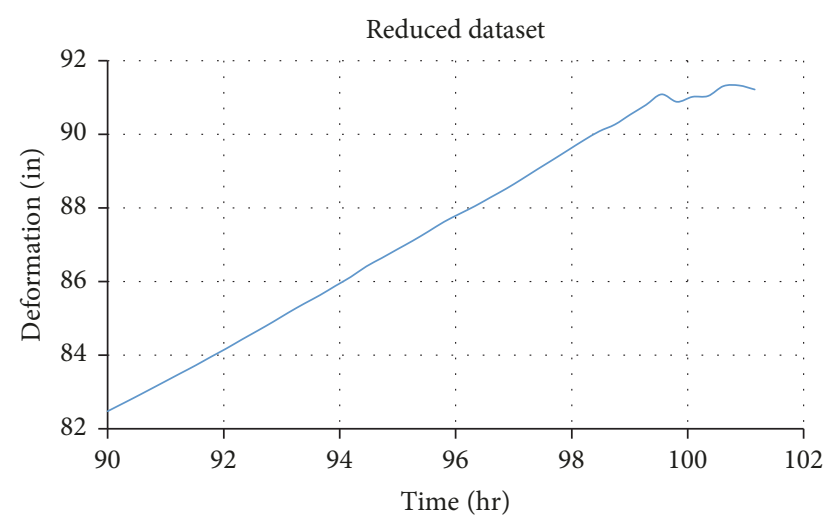

(b) Reduced dataset shows linear trend

Figure 9

and the size of the area to cover, operating the monitoring equipment at high scan rates may not be feasible.

Assessing the potential for failure based on the traditional inverse velocity and the minimum inverse velocity method proposed by the authors in a previous publication depends to a large extent on establishing an appropriate trend line. A velocity curve that is noisy or presents a strong bias effectively limits the potential for making a reliable prediction. The ML method, however, is less prone to noise and bias because it uses time-deformation data. Velocity is calculated by differentiating the deformation curve. Inherently, the process of differentiation amplifies higher frequencies in the time series, hence the requirement to smooth out the velocity data before using the inverse velocity or minimum inverse velocity techniques.

The analysis presented in Table 5 provides another opportunity to quantitatively assess the imminence of failure using the ML method. When creating the training set, one of the requirements is to align the datasets with respect to the actual time of failure in each set. In this study, the training set contained data from eight hours prior to failure. As expected, the prediction network performed best when a multistep prediction was carried out on a test set with a one- and twohour window of missing data prior to failure. In this case, the peak in the prediction curve aligns closely with the peaks in the training set. However, when a test set with a time window far from the actual time of failure is used, a significant shift in the failure peak of the predicted time series is observed. This measure could be used to further verify the accuracy of predictions.

A progressive trend in the deformation data would be most concerning as it has a higher probability of failure. The seven datasets in Table 3 with a difference of more than 30 minutes from the actual time of failure did not show a smooth progressive trend. Datasets that contain a high amount of atmospheric noise (due to rain, wind, or dust) or large movement resulting from mining activity could adversely affect the predicted values. When a slope has been moving for an extended period of time, or if the movement is accelerating, the deformation data would show a linear trend as it moves away from the inflection point that marks the beginning of a progressive curve.

For ML to perform well, all the datasets are required to be of the same length. One could reason that this is a drawback of the ML method. For all the datasets to be of the same length, it might be necessary to shorten the length of the data from an area that has been moving for an extended period of time. Shortening a dataset may remove the inflection point that marks the beginning of a progressive trend. If the data does not include the inflection point, the deformation curve will tend, in general, to show a linear trend line instead of a progressive trend. If the training set does not contain strong progressive features, predictions on the test set with a linear trend could lead to inaccurate failure predictions. The deformation curve from mine site 7 is an example of a dataset that has a progressive trend; however, the data in the eighthour window before the slope failure displays a linear trend (Figures 9(a) and 9(b)).

To improve the performance of the prediction network, it is therefore recommended to include in the training set deformation data that show similar behavior in terms of the degree of curvature around the inflection point. If a large dataset is available, several training sets could be created based on a judicious grouping of curvature indexes calculated for each time-deformation curve.

\section{Conclusion}

Geotechnical risk management analysis is the key to successfully manage the risks posed to personnel, equipment, and production at an active mine [6]. Slope failures have been an issue in the past and continue to be a threat today. To effectively mitigate the risks of unstable slopes, it is important to make more reliable predictions. Slope failure predictions are only helpful when they allow sufficient time to remove people and equipment from the unsafe areas. As all mining operations are different, they may have different escape routes planned in case of emergencies. For this study, the term ample evacuation time is tied together with a safe prediction. It is assumed that each mine will have an estimated required 
evacuation time based on the size of the mine and the resources available. For the purpose of this study, it is believed that if the predicted time of failure is very close to the real failure or before the collapse, it will provide sufficient time for evacuation. In other words, we want to make a time of failure prediction that gives us the confidence to evacuate if required. For example, if a mine requires 2 hours of evacuation time and the failure was predicted to happen in the next 4 hours, it would be necessary to evacuate the mine 2 hours after the prediction was made or 2 hours before the expected time of failure. It is important to understand that no predetermined amount of time can be considered as ample evacuation time as it can vary from one mine to another and it can also vary between different sections of a single mine.

The current study proposed the use of machine learning (ML) to predict the time of failure. The results of the study show that ML provided prediction values that are $86 \%$ of the time closer to the actual time of failure when compared to the traditional IV method. When compared to MIV, ML had a $72 \%$ success rate. All the failure predictions using ML method were within 2 hours of the actual time of failure. Fifteen out of the 22 datasets analyzed gave a time of failure prediction that was within 30 minutes of the actual time of failure. Only 2 datasets gave a failure prediction that was over 60 minutes away from the real time of failure. The ML method resulted in 17 datasets with safe predictions and only 5 sets with an unsafe prediction. The five sets with an unsafe prediction were within 5 minutes of the actual failure time, making the unsafe predictions reliable. A larger training set containing carefully selected data based on the similarity of the deformation curves would further improve the reliability of slope failure predictions.

\section{Conflicts of Interest}

The authors declare that they have no conflicts of interest.

\section{References}

[1] K. S. Osasan and T. R. Stacey, "Automatic prediction of time to failure of open pit mine slopes based on radar monitoring and inverse velocity method," International Journal of Mining Science and Technology, vol. 24, no. 2, pp. 275-280, 2014.

[2] L. Nie, Z. Li, Y. Lv, and H. Wang, "A new prediction model for rock slope failure time: a case study in West Open-Pit mine, Fushun, China," Bulletin of Engineering Geology and the Environment, vol. 76, no. 3, pp. 975-988, 2016.

[3] H. Chen, Z. Zeng, and H. Tang, "Landslide deformation prediction based on recurrent neural network," Neural Processing Letters, vol. 41, no. 2, pp. 169-178, 2015.

[4] Z. Liu, J. Shao, W. Xu, H. Chen, and C. Shi, "Comparison on landslide nonlinear displacement analysis and prediction with computational intelligence approaches," Landslides, vol. 11, no. 5, pp. 889-896, 2014.

[5] P. Mazzanti, F. Bozzano, and I. Cipriani, "New insights into the temporal prediction of landslides by a terrestrial SAR interferometry monitoring case study," Landslides, vol. 12, no. 1, pp. 55-68, 2015.

[6] U. P. Chandarana, M. Momayez, and K. Taylor, "Monitoring and predicting slope instability: a review of current practices from a mining perspective," International Journal of Research in Engineering and Technology, vol. 5, no. 11, pp. 139-151, 2016.

[7] A. Federico, M. Popescu, G. Elia, C. Fidelibus, G. Internò, and A. Murianni, "Prediction of time to slope failure: a general framework," Environmental Earth Sciences, vol. 66, no. 1, pp. 245-256, 2012.

[8] G. B. Crosta and F. Agliardi, "Failure forecast for large rock slides by surface displacement measurements," Canadian Geotechnical Journal, vol. 40, no. 1, pp. 176-191, 2003.

[9] M. Saito, "Forecasting time of slope failure by tertiary creep," in Proceedings of the Proceedings of the 7th International Conference on Soil Mechanics and Foundation Engineering, pp. 677-683, 1996.

[10] Q. Xu, Y. Yuan, and Y. Zeng, "Some new pre-warning criteria for creep slope failure," Science China Technological Sciences, vol. 54, no. 1, pp. 210-220, 2011.

[11] T. Fukuzono, "A new method for predicting the failure time of a slope," in Proceedings of the Fourth International Conference and Field Workshop on Landslides, pp. 150-150, Japan Landslide Society, Tokyo, Japan, 1985.

[12] T. Mitchell, Machine Learning, Science/Engineering/Math, McGraw-Hill, New York, NY, USA, 1997.

[13] M. Paluszek and S. Thomas, MATLAB Machine Learning, vol. 1, 2017.

[14] S. H. Ni, P. C. Lu, and C. H. Juang, "Fuzzy neural network approach to evaluation of slope failure potential," Microcomputers in Civil Engineering, vol. 11, pp. 56-66, 1996.

[15] M. G. Sakellariou and M. D. Ferentinou, "A study of slope stability prediction using neural networks," Geotechnical and Geological Engineering, vol. 23, no. 4, pp. 419-445, 2005.

[16] H. B. Wang, W. Y. Xu, and R. C. Xu, "Slope stability evaluation using Back Propagation Neural Networks," Engineering Geology, vol. 80, no. 3-4, pp. 302-315, 2005.

[17] S. Hwang, I. F. Guevarra, and B. Yu, "Slope failure prediction using a decision tree: a case of engineered slopes in South Korea," Engineering Geology, vol. 104, no. 1-2, pp. 126-134, 2009.

[18] H.-M. Lin, S.-K. Chang, J.-H. Wu, and C. H. Juang, "Neural network-based model for assessing failure potential of highway slopes in the Alishan, Taiwan Area: pre- and post-earthquake investigation," Engineering Geology, vol. 104, no. 3-4, pp. 280289, 2009.

[19] L. A. Zadeh, "Fuzzy sets," Information and Control, vol. 8, no. 3, pp. 338-353, 1965.

[20] U. C. Kothari and M. Momayez, "New approaches to monitoring, analyzing and predicting slope instabilities," Journal of Geology and Mining Research, vol. 1, pp. 1-14, 10. 

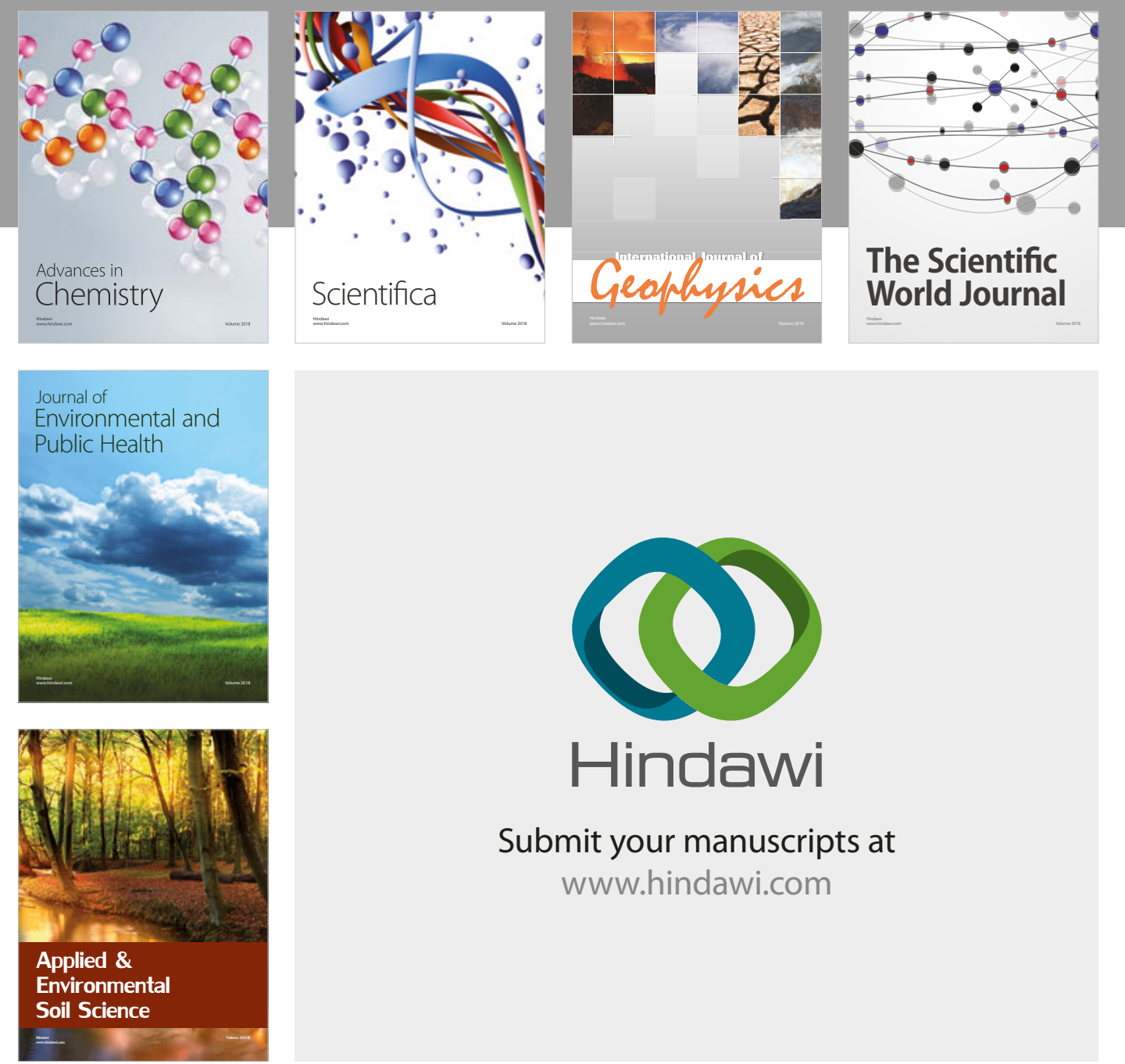

The Scientific

\section{World Journal}
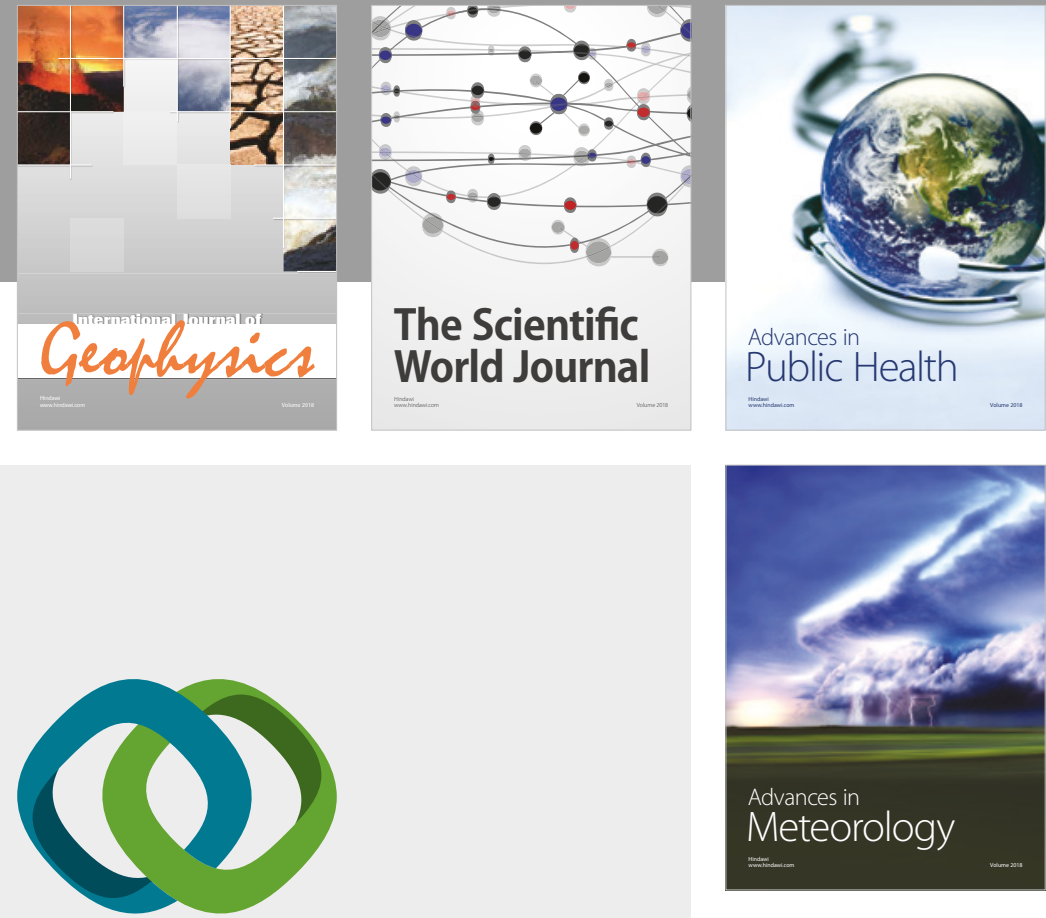

Advan

Public Health

\section{Hindawi}

Submit your manuscripts at

www.hindawi.com
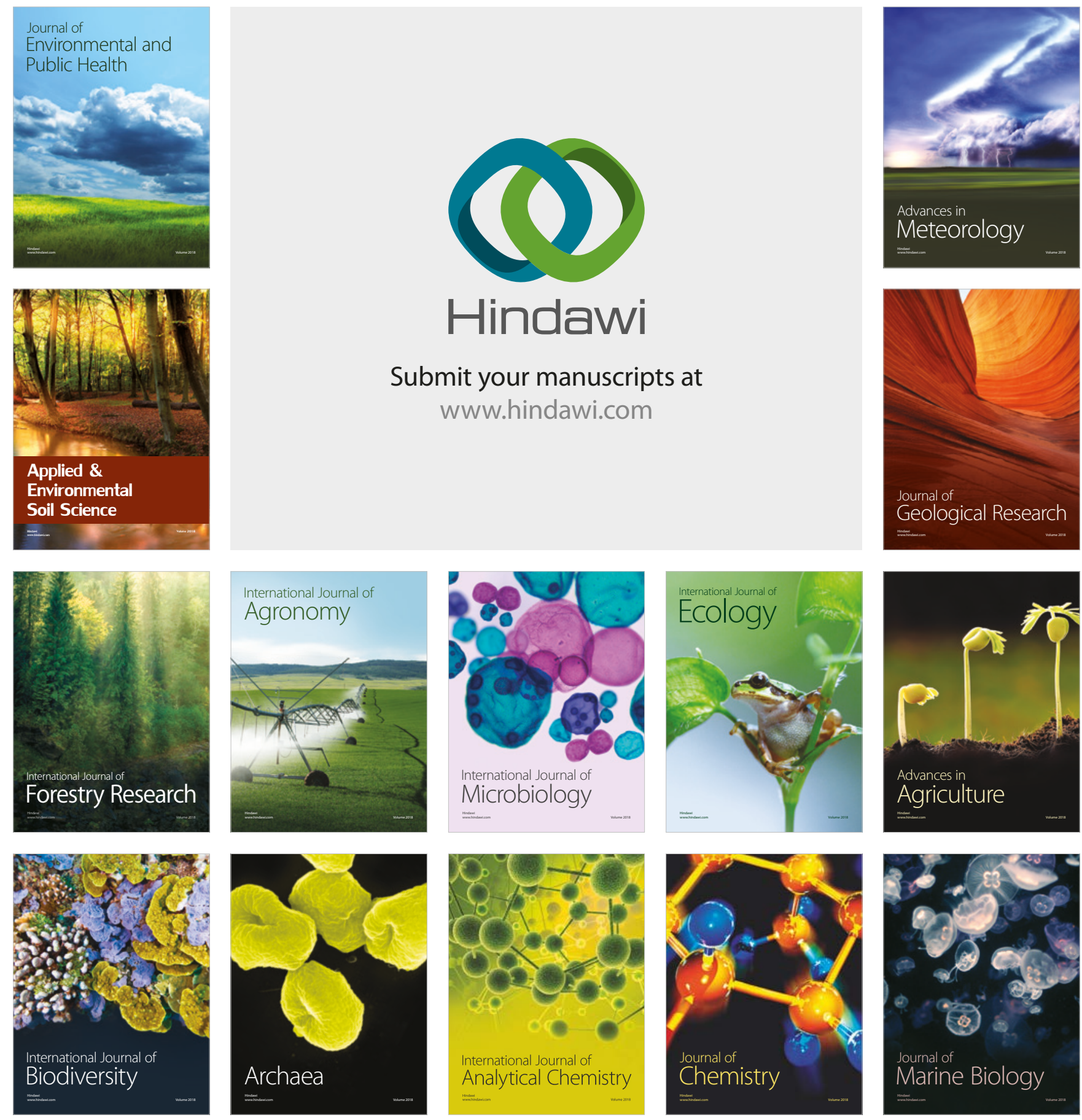